\title{
A Novel Radial-Composite Model of Pressure Transient Analysis for Multistage Fracturing Horizontal Wells with Stimulated Reservoir Volume
}

\author{
Yunpeng Hu $\mathbb{D}^{\mathbb{D}}$, Xiaoling Zhang $\mathbb{D}^{\mathbb{D}}$, Ziyun Cheng $(\mathbb{D}$, Wei Ding $\mathbb{D}$, Liangchao Qu $(\mathbb{D}$, \\ Penghui Su, Chunliu Sun $\mathbb{D}$, and Wenqi Zhang $\mathbb{D}$
}

PetroChina Research Institute of Petroleum Exploration and Development, Beijing, China

Correspondence should be addressed to Yunpeng Hu; huyunpeng1129@petrochina.com.cn

Received 5 November 2020; Revised 7 December 2020; Accepted 21 December 2020; Published 23 January 2021

Academic Editor: Lei Wang

Copyright (c) 2021 Yunpeng Hu et al. This is an open access article distributed under the Creative Commons Attribution License, which permits unrestricted use, distribution, and reproduction in any medium, provided the original work is properly cited.

\begin{abstract}
In the process of stimulated reservoir volume of tight reservoir, horizontal well can form three zones, the inner zone is multistage fracturing zone, the middle zone is skin damage zone, and the outer zone is undamaged zone. In this paper, a transient well test analysis model of multistage fracturing horizontal well in three area composite reservoir is proposed. Based on Laplace transformation, point source integration, and superposition principle, the infinite conductivity multifracture model of three area composite reservoir is obtained. The linear equations of finite conductivity multifracture in Laplace space are established by using the equal conditions of flow and pressure at the fracture wall. Gauss-Newton iteration method and Stehfest number are used to obtain the solution of wellbore pressure. The accuracy of the results is verified by numerical simulation. Then, the flow characteristics of multistage fracturing horizontal wells in three area composite reservoirs are analyzed by type curves. The flow is divided into ten stages, which are the bilinear flow, the linear flow, the first radial flow stage, the inner zone linear flow, the inner zone radial flow, the middle zone linear flow, the middle zone radial flow, the outer zone linear flow, the outer zone radial flow, and the boundary dominated flow. The pressure derivative curves show different characteristics in different flow stages. The influences of fracture conductivity, fracture spacing, radius ratio of the middle zone to inner zone, radius ratio of the outer zone to the middle zone, permeability ratio of inner zone to the middle zone, permeability ratio of inner zone to outer zone, storage capacity ratio of inner zone to the middle zone, and storage capacity ratio of inner zone to outer zone on type curves are analyzed. Finally, the application and reliability of the proposed model are verified by a case example.
\end{abstract}

\section{Introduction}

China is rich in unconventional oil and gas resources with great development potential. The efficient development of unconventional oil and gas resources is of great significance to meeting China's energy needs [1]. With the continuous advancement of hydraulic fracturing technologies, horizontal wells are generally subjected to multistage perforating and fracturing to increase the drainage area of reservoirs, improve the development effect of reservoirs and the connectedness of fluids between reservoirs and wellbore, and then increase the production of single wells [2]. The analysis of pressure dynamic characteristics is an important method for evaluating reservoirs and obtain- ing physical property parameters of reservoirs and is of great significance to providing guidance to the development of oil and gas fields.

Many scholars have made remarkable achievements in the simulation of pressure and the dynamic analysis of productivity of vertical fractured wells. In 1973, Gringarten et al. introduced the point source function to seepage mechanics and solved the infinite formation seepage equation by means of the point source function and Green function. In addition, they analyzed infinitely homogeneous reservoirs, double porosity media, fractures with infinite conductivity, and dynamic characteristics of well pressure [3, 4]. In 1978, Cinco-Ley et al. firstly proposed the concept of "bilinear flow." According to the research 
results of Cinco-Ley et al., fractures in fractured wells have finite conductivity and the seepage in fractured wells can be divided into four stages. Cinco-Ley et al. solved the pressure solution for each flow stage [5, 6]. Later, many scholars did a lot of improvement work based on Cinco's model and applied their results in the well test analysis of multistage fractured horizontal wells [7-9].

Due to the uneven development of lithology and volume fracturing, low permeability reservoirs have planar heterogeneity and tend to show the characteristics of composite reservoirs. Ozkan et al. [10] considered the "volume fracturing" characteristics of hydraulic fractures in tight reservoirs and represented the region between fractures as a double-porosity medium. In addition, they built a new unsteady-state productivity analysis model for low permeability homogeneous medium in other parts. Brohi et al. [11] established a two-region double-porosity medium composite model, considering the influence of hydraulic fractures on regional permeability changes. Ozkan et al. [10] discussed the difference in production performance of fractured horizontal wells in conventional and unconventional reservoirs using a trilinear percolation model. Stalgorova and Mattar [12] combined with Brown et al. [13] to extend the model to a five-linear seepage model. Wang et al. [14] considered the difference in physical properties of reservoirs between stimulated zones and nonstimulated zones caused by volume fracturing in tight reservoirs and quantitatively investigated the fluid channeling between different zones. Tao et al. [15] presented a new productivity prediction hybrid based on grey relation analysis. Ahmadi et al. [16] analyzed the multifractured well production data using the dynamic drainage area concept.

The above authors all considered planar heterogeneity caused by volume fracturing. The interpretation and processing results of a large number of site well test data indicate that many oil wells often show changes in physical properties of reservoirs around their wellbore or at a certain distance away from the wellbore and are characterized by three-region or multiregion composite reservoirs. A horizontal well can be divided into three regions in the process of volume fracturing stimulation: the inner region is a multistage fracturing region, the middle region is a skin damage region, and the outer region is a nonstimulated region. Based on this, the purpose of the paper is to establish a new well test analysis model for multistage fractured horizontal wells in a three-region composite reservoir. In this paper, a point source seepage model for a three-region composite reservoir has been firstly established; moreover, an infinite conductivity multifracture solution to the three-region composite reservoir has been obtained by means of superposition principle and point source integration. Then, the linear equations of fracture flow rate and horizontal well pressure in Laplace space have been obtained by coupling the Fredholm integral equation, and a real space pressure solution has been obtained using the Stehfest numerical inversion algorithm. Finally, the typical curves and flow characteristics have been analyzed using the obtained solution, thereby, estab- lishing a theoretical foundation for the well test analysis of multistage fractured horizontal wells in the threeregion composite reservoir.

\section{Establishment of New Models for Multistage Fractured Horizontal Wells in a Multiregion Composite Reservoir}

2.1. Physical Model. Figures 1(a) and 1(b) are the schematic diagram of the model for the multistage fractured horizontal well in the three-region composite reservoir. After multistage fracturing of the horizontal well in the tight reservoir, multistage fractures are formed in the well (Figure 1(b)). After volume fracturing of the multistage fractured horizontal well, the reservoir is divided into three regions. The first region is the fractured region formed first near the wellbore after the forming of the stimulated region near the bottom hole through fracturing; the second region is a skin damage region; the third region is an undamaged region. For convenience, the three regions are called the inner region, middle region, and outer region. Fluids flow first from the outer region to the middle region, then from the middle region to the inner region, from the inner region to the hydraulic fractures, and finally from the hydraulic fractures to the wellbore (Figure 2). The physical model is simplified below:

(1) The three-region reservoir is divided into inner region, middle region, and outer region, and their permeability is $k_{1}, k_{2}$, and $k_{3}$, respectively

(2) All rock properties such as reservoir permeability, reservoir porosity, hydraulic fracture permeability, and hydraulic fracture porosity are all a constant. The compressibility factor and viscosity of fluids and reservoir thickness are also a constant

(3) The hydraulic fracture formed by staged fracturing of the horizontal well is assumed to run through the whole reservoir; in addition, the length of each fracture is $x_{f}$ and the fracture width is $w$ on $x-y$ plane

(4) The reservoir belongs to the radial coordinate; the horizontal and radial outer boundaries of the reservoir are closed, and the upper and lower boundaries are impermeable

(5) Isothermal Darcy seepage occurs in the reservoir; moreover, consider the occurrence of finite conductivity flow in the hydraulic fractures

(6) The total production of all hydraulic fractures is a constant $Q$, so the pressure changes with time

(7) The pressure is distributed uniformly in initial state and equals to the initial pressure $P_{i}$

2.2. Point Source Model for Three-Region Porous Medium Enclosed Space. According to the assumptions of the 


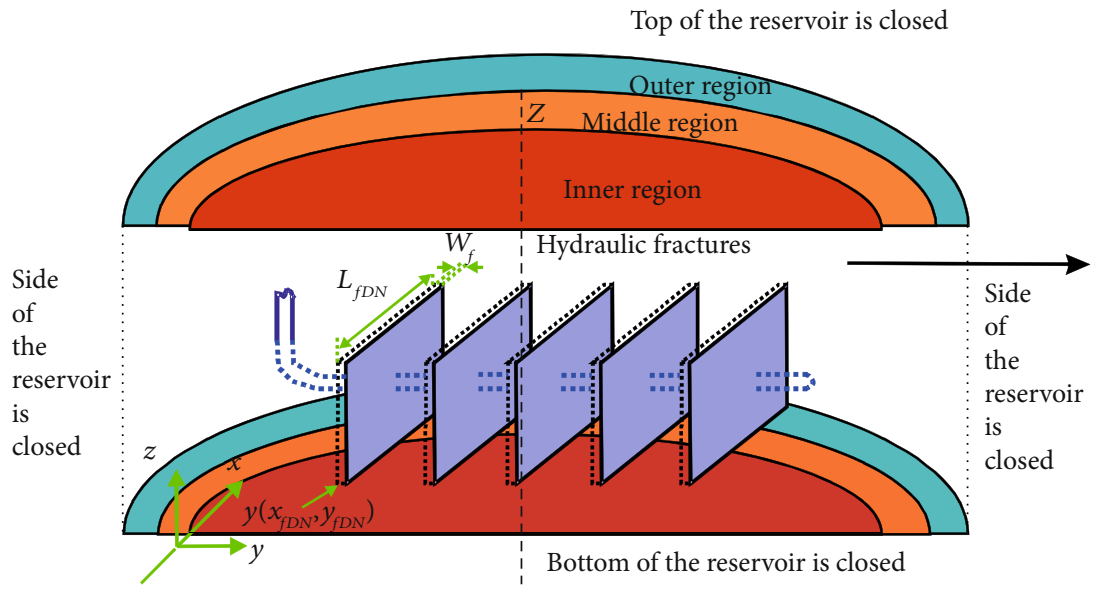

(a)

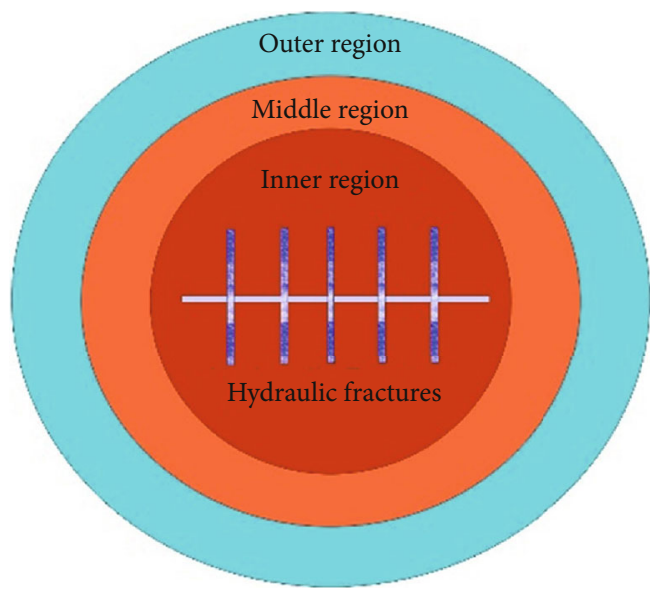

(b)

FIGURE 1: Schematic of the multistage fractured horizontal well in the three-region heterogeneous reservoir, where the inner region is the volume fracturing stimulation region of the multistage fractured horizontal well, the middle region is the skin damage region, and the outer region is the nonstimulated and undamaged region. ((a) Multiregion heterogeneous space schematic; (b) multiregion heterogeneous plane schematic).

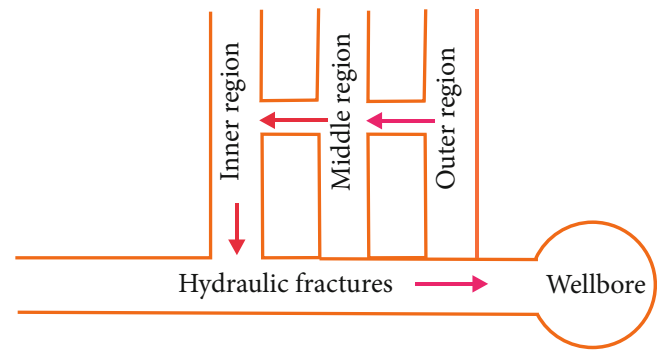

FIGURE 2: Schematic of flow in the multistage fractured horizontal well in the three-region reservoir.

physical model, it is known that the reservoir is divided into three regions through multistage fracturing stimulation of the horizontal wells. The inner region is the volume fracturing stimulation region; the middle region is the skin damage region, and the outer region is the nonstimulated and undamaged region. By combining the continuity equation, velocity equation, and state equation, the obtained system of equations for seepage control in dual permeability medium is as follows (for the following symbols, see their annotations):

$$
\left\{\begin{array}{l}
\frac{1}{r} \frac{\partial}{\partial r}\left(r \frac{\partial p_{1}}{\partial r}\right)=\frac{\phi_{1} \mu c_{t 1}}{k_{1}} \frac{\partial p_{1}}{\partial t}, \\
\frac{1}{r} \frac{\partial}{\partial r}\left(r \frac{\partial p_{2}}{\partial r}\right)=\frac{\phi_{2} \mu c_{t 2}}{k_{2}} \frac{\partial p_{2}}{\partial t} \\
\frac{1}{r} \frac{\partial}{\partial r}\left(r \frac{\partial p_{3}}{\partial r}\right)=\frac{\phi_{3} \mu c_{t 3}}{k_{3}} \frac{\partial p_{3}}{\partial t}
\end{array} .\right.
$$

Internal boundary constant flow rate:

$$
\left[2 \pi r h \frac{k_{1}}{\mu} \frac{\partial p_{1}}{\partial r}\right]_{r \rightarrow 0}=Q .
$$


There are pressure and flow connection conditions at the interfaces between the inner region and the middle region and between the middle region and the outer region:

$$
\left\{\begin{array}{l}
p_{1}=\left.p_{2}\right|_{r=r_{e 1}}, \\
p_{2}=\left.p_{3}\right|_{r=r_{e 1}}, \\
\frac{k_{1}}{\mu} \frac{\partial p_{1}}{\partial r}=\left.\frac{k_{2}}{\mu} \frac{\partial p_{2}}{\partial r}\right|_{r=r_{e 1}}, \\
\frac{k_{2}}{\mu} \frac{\partial p_{2}}{\partial r}=\left.\frac{k_{3}}{\mu} \frac{\partial p_{3}}{\partial r}\right|_{r=r_{e 2}} .
\end{array} .\right.
$$

The internal boundary is assumed to be closed:

$$
\left.\frac{k_{3}}{\mu} \frac{\partial p_{3}}{\partial r}\right|_{r=r_{e 3}}=0 .
$$

The following dimensionless quantities are defined for convenience of the solution:

$$
\begin{aligned}
& r_{D}=\frac{r}{x_{f}} \lambda_{21}=\frac{k_{1}}{k_{2}} \lambda_{31}=\frac{k_{1}}{k_{3}} \omega_{21}=\frac{\left(\phi \mu c_{t}\right)_{1}}{\left(\phi \mu c_{t}\right)_{3}} \omega_{31}=\frac{\left(\phi \mu c_{t}\right)_{1}}{\left(\phi \mu c_{t}\right)_{3}}, \\
& p_{1 D}=\frac{2 \pi k_{1} h}{Q \mu}\left(p_{i}-p_{1}\right) p_{2 D}=\frac{2 \pi k_{1} h}{Q \mu}\left(p_{i}-p_{22}\right) p_{3 D}=\frac{2 \pi k_{1} h}{Q \mu}\left(p_{i}-p_{3}\right), \\
& r_{e D 1}=\frac{r_{e 1}}{x_{f}} r_{e D 2}=\frac{r_{e 2}}{k_{2}} r_{e D 3}=\frac{r_{e 3}}{x_{f}} t_{\Delta_{21}=\omega_{21} / \lambda_{21} \Delta_{31}=\omega_{31} / \lambda_{31} .} t_{D}=\frac{k_{1} t}{\left(\phi c_{t}\right)_{1} \mu x_{f}^{2}} .
\end{aligned}
$$

According to the above dimensionless definitions, equations (1) to (4) can be transformed into the following dimensionless form:

$$
\left\{\begin{array}{l}
\frac{\partial^{2} p_{1 D}}{\partial r_{D}^{2}}+\frac{1}{r} \frac{\partial^{2} p_{1 D}}{\partial r_{D}}=\frac{\partial p_{1 D}}{\partial t_{D}} \\
\frac{\partial^{2} p_{2 D}}{\partial r_{D}^{2}}+\frac{1}{r} \frac{\partial p_{2 D}}{\partial r_{D}}=\frac{1}{\Delta_{21}} \frac{\partial p_{2 D}}{\partial t_{D}} \\
\frac{\partial^{2} p_{3 D}}{\partial r_{D}^{2}}+\frac{1}{r} \frac{\partial p_{3 D}}{\partial r_{D}}=\frac{1}{\Delta_{31}} \frac{\partial p_{3 D}}{\partial t_{D}}
\end{array}\right.
$$

The internal and external boundary conditions are transformed into:

$$
\left\{\begin{array}{l}
\left.r_{D} \frac{\partial p_{1 D}}{\partial r_{D}}\right|_{r_{D}=0}=-q_{D}(s), \\
p_{1 D}=\left.p_{2 D}\right|_{r_{D}=r_{C D 1}} \\
p_{2 D}=\left.p_{3 D}\right|_{r_{D}=r_{e D 2}} \\
\frac{\partial p_{1 D}}{\partial r_{D}}=\frac{1}{\lambda_{21}} \frac{\partial p_{2 D}}{\partial r_{D}}, \\
\frac{1}{\lambda_{21}} \frac{\partial p_{2 D}}{\partial r_{D}}=\frac{1}{\lambda_{31}} \frac{\partial p_{3 D}}{\partial r_{D}}, \\
\left.\frac{\partial p_{3 D}}{\partial r_{D}}\right|_{r_{D}=r_{e D 3}}=0 .
\end{array}\right.
$$

Through Laplace transformation, the general solution of the above equations is

$$
\begin{gathered}
\tilde{p}_{1 D}=\tilde{q}_{D}(s) \cdot\left[K_{0}\left(r_{D} \sqrt{s}\right)+B_{5} I_{0}\left(r_{D} \sqrt{s}\right)\right], \\
\tilde{p}_{2 D}=A_{3} K_{0}\left(r_{D} \sqrt{s / \Delta_{21}}\right)+A_{4} I_{0}\left(r_{D} \sqrt{s / \Delta_{21}}\right), \\
\tilde{p}_{3 D}=A_{5} K_{0}\left(r_{D} \sqrt{s / \Delta_{31}}\right)+A_{6} I_{0}\left(r_{D} \sqrt{s / \Delta_{31}}\right) .
\end{gathered}
$$

In the above equations: $A_{1}=1 / s ; A_{2}=A_{1} \cdot B_{5}$;

$$
\begin{gathered}
B_{5}=\frac{B_{4} K_{1}\left(r_{e D 1} \sqrt{s}\right)-K_{0}\left(r_{e D 1} \sqrt{s}\right)}{B_{4} I_{1}\left(r_{e D 1} \sqrt{s}\right)-I_{0}\left(r_{e D 1} \sqrt{s}\right)}, \\
B_{4}=\frac{K_{0}\left(r_{e D 1} \sqrt{s / \Delta_{31}}\right)+B_{3} I_{0}\left(r_{e D 1} \sqrt{s / \Delta_{31}}\right)}{\left(1 / \lambda_{21} \sqrt{\Delta_{21}}\right)\left(K_{1}\left(r_{e D 1} \sqrt{s / \Delta_{31}}\right)-B_{3} I_{0}\left(r_{e D 1} \sqrt{s / \Delta_{31}}\right)\right)}, \\
B_{2}=\frac{B_{2} K_{1}\left(r_{e D 2} \sqrt{s / \Delta_{21}}-K_{0}\left(r_{e D 2} \sqrt{s / \Delta_{21}}\right)\right)}{I_{0}\left(r_{e D 2} \sqrt{s / \Delta_{21}}+B_{2} I_{1}\left(r_{e D 2} \sqrt{s / \Delta_{21}}\right)\right)}, \\
\left.\lambda_{21} / \lambda_{31}\right) \sqrt{\left(\Delta_{21} / \Delta_{31}\right)\left(K_{e D 2} \sqrt{s / \Delta_{31}}+B_{1} I_{0}\left(r_{e D 2} \sqrt{s / \Delta_{31}}\right)-B_{1} I_{1}\left(r_{e D 2} \sqrt{s / \Delta_{31}}\right)\right)}
\end{gathered}
$$

Taking fracture half-length as the reference length, the dimensionless length of the fracture is 1 . The position of any point of the reservoir and the point source is, respectively $\left(x_{D}, y_{D}\right)$ and $\left(x_{D}, y_{D}{ }^{\prime}\right)$, and the distance of the point source to any point is $r_{D}$. Then, the following geometrical relationship is established:

$$
r_{D}^{2}=\left(x_{D}-x_{D}{ }^{\prime}\right)^{2}+\left(y_{D}-y_{D}{ }^{\prime}\right)^{2} .
$$


The hydraulic fracture is located in the stimulation region, so the formula used for point source integration is the inner region pressure formula. Substitute equation (16) into the inner region pressure formula in equation (10) and then obtain:

$$
\tilde{p}_{1 D}=\tilde{q}_{D}(s) \cdot \xi\left(x_{D}, y_{D}, x_{D}^{\prime}, y_{D}^{\prime}, r_{e D 1}, r_{e D 2}, r_{e D 3}, s\right)
$$

where

$\xi\left(x_{D}, y_{D}, x_{D}^{\prime}, y_{D}^{\prime}, r_{e D 1}, r_{e D 2}, r_{e D 3}, s\right)=\left[K_{0}\left(r_{D} \sqrt{s}\right)+B_{5} I_{0}\left(r_{D} \sqrt{s}\right)\right]$,

where $s$ is Laplace space time variable. Assuming that the number of fractures in the multistage fractured horizontal well is $N_{f}$, the pressure drop of each section of fractures can be obtained as follows by means of point source integration and superposition principle:

$\tilde{p}_{D j}=\sum_{j=1}^{N_{f}} \int_{0}^{1} \tilde{q}_{f D j}\left(\gamma_{D j}, s\right) \xi\left(x_{D j}, y_{D j}, x_{D}^{\prime}, y_{D}^{\prime}, r_{e D 1}, r_{e D 2}, r_{e D 3}, s\right) d L$,

where $d L=\sqrt{d x^{2}+d y^{2}}=d x \sqrt{1+\tan ^{2} \theta}$ is the integration path, and $\theta$ is the included angle with the $y$-axis. ( $\left.x_{D j}, y_{D j}\right)$ is the coordinate of the center point of the $j^{\text {th }}$ fracture, and $q_{\mathrm{fDj}}$ is the flow rate of the $j^{\text {th }}$ fracture. If each fracture is divided into $N_{\text {sj }}$ sections, the pressure drop formula for the $i^{\text {th }}$ section of the $j^{\text {th }}$ fracture is as follows:

$\tilde{p}_{D j i}=\sum_{m=1}^{N_{f}} \sum_{n=1}^{N_{s m}} \tilde{q}_{f D m n} \int_{(n-1) \Delta x}^{n \Delta x} \xi\left(x_{D j i}, y_{D j i}, x_{D}^{\prime}, y_{D}^{\prime}, r_{e D 1}, r_{e D 2}, r_{e D 3}, s\right) d L$,

where $\Delta x=1 / N_{s m}$ is the hydraulic fracture section length. $\left(x_{D j i}, y_{D j i}\right)$ is the coordinate of the center point of the $i^{\text {th }}$ section of the $j^{\text {th }}$ fracture. Where $k_{1}$ is the permeability of the inner region, $\mathrm{m}^{2} ; k_{2}$ is the permeability of the middle region, $\mathrm{m}^{2} ; k_{3}$ is the permeability of the outer region, $\mathrm{m}^{2} ; r_{e 1}$ is the radius of the inner region, $\mathrm{m} ; r_{e 2}$ is the radius of the middle region, $\mathrm{m} ; r_{e 3}$ is the radius of the outer region, $\mathrm{m} ; \varphi_{1}, \varphi_{2}$, and $\varphi_{3}$ are the porosity of the inner region, middle region, and outer region, respectively; $c_{t 1}, c_{t 2}$, and $c_{t 3}$ are the total compressibility factor of the inner region, middle region, and outer region, respectively, $1 / \mathrm{Pa} ; \mu$ is oil viscosity, $\mathrm{cp} ; t$ is time, $\mathrm{s} ; r$ is radial radius, $m ; \lambda_{21}$ and $\lambda_{31}$ are the permeability ratio of the inner region to the middle region and the permeability ratio of the inner region to the outer region, respectively; $\omega_{21}$ and $\omega_{31}$ are the storage ratio of the inner region to the middle region and the storage ratio of the inner region to the outer region, respectively; $p_{1}, p_{2}$, and $p_{3}$ are the pressure of the inner region, middle region, and outer region, respectively, $\mathrm{Pa} ; x_{f}$ is fracture half-length, $\mathrm{m} ; K_{0}$ is the zero-order modified Bessel function; $Q$ is horizontal well production, $\mathrm{m}^{3} / \mathrm{s} ; D$ is a dimensionless subscript.

2.3. Hydraulic Fracture Flow Model. The flow in fractures can be assumed to be one-dimensional linear flow. According to the idea of Cinco-Ley (1988), the dimensionless governing equation of flow in the single wing of a one-dimensional fracture can be written as follows:

$$
-\frac{\partial^{2} p_{f D}}{\partial r_{D}}+\frac{2 \pi}{C_{f D}}=0
$$

The initial condition of a hydraulic fracture can be written as:

$$
p_{f D}\left(r_{D}, t_{D}=0\right)=0,0 \leq r_{D} \leq 1
$$

The internal boundary condition can be written as:

$$
\begin{gathered}
\left(\frac{\partial p_{f D}}{\partial r_{D}}\right)_{r_{D}=0}=-\frac{2 \pi}{C_{f D}} q_{f w D}, \\
\left(\frac{\partial p_{f D}}{\partial r_{D}}\right)_{r_{D}=1}=0 .
\end{gathered}
$$

Perform double integration of equation (21) from 0 to $v$ and from 0 to $\mathrm{rD}$ while using boundary equations (24) and (25) to obtain:

$$
p_{f D}\left(r_{D}\right)=p_{w D}+\frac{2 \pi}{C_{f D}} \int_{0}^{r_{D}} \int_{0}^{v} q_{f D}(u) d u d v-\frac{2 \pi}{C_{f D}} q_{f w D} .
$$

Carry out Laplace transformation to obtain:

$$
\tilde{p}_{f D}\left(r_{D}\right)=\tilde{p}_{w D}+\frac{2 \pi}{C_{f D}} \int_{0}^{r_{D}} \int_{0}^{v} \tilde{q}_{f D}(u) d u d v-\frac{2 \pi}{C_{f D}} \tilde{q}_{f w D} .
$$

Assume that a multistage fractured horizontal well consists of multiple single-wing fractures. If the $j^{\text {th }}$ fracture wing is divided into $n_{j}$ sections, the pressure of the $i^{\text {th }}$ section of fracture can be obtained from the discrete equation (30):

$$
\begin{aligned}
\tilde{p}_{f D}\left(r_{D}\right) & =\tilde{p}_{w D}-\frac{2 \pi}{C_{f D}} \cdot\left\{\left(i \Delta r_{D}-\frac{\Delta r_{D}}{2}\right) \cdot \sum_{k=1}^{n_{j}} q_{f D k}-\frac{\Delta r_{D}}{8}\right. \\
& \left.\cdot\left(q_{f D i}\right)-\sum_{k=1}^{i-1}\left(q_{f D i}\right) \cdot\left[\frac{\Delta r_{D}}{2}+\left(i \Delta r_{D}-\left(k+\frac{1}{2}\right) \Delta r_{D}\right)\right]\right\}
\end{aligned}
$$

where $\Delta r_{D}=1 / n_{j} ; \tilde{p}_{f D i}$ and $\tilde{q}_{f D i}$ are the pressure and flow rate of the $i^{\text {th }}$ section of the $j^{\text {th }}$ fracture wing. $C_{\mathrm{fD}}$ is dimensionless fracture conductivity. 
2.4. Coupling Solution to the Multiregion Reservoir Model and Fracture Model. Assume that $N$ fracture wings are formed during horizontal well fracturing. If the $j^{\text {th }}$ fracture wing is divided into $n_{j}$ sections, the total number of fracture sections is

$$
m=\sum_{j=1}^{N} n_{j}
$$

Based on the superposition principle, the pressure on the fracture surface shall be equal to that on the hydraulic fracture section, then,

$$
\tilde{p}_{f D j i}=\sum_{k=1}^{N} \sum_{m=1}^{n_{k}} \tilde{q}_{f D k m} \cdot s \tilde{p}_{D(j i, k m)},
$$

where the subscript $j i$ is the $i^{\text {th }}$ section of the $j^{\text {th }}$ fracture wing; $\tilde{p}_{D(j i, k m)}$ is the influence of the $m^{\text {th }}$ section of the $k^{\text {th }}$ fracture on the pressure drop in the $i^{\text {th }}$ section of the $j^{\text {th }}$ fracture. Now $m$ equations can be listed, but there are $m+1$ unknowns. Assuming that the horizontal well is produced at a fixed flow rate, the sum of the flow rates of all fracture sections shall be equal to the fixed flow rate of the horizontal well; then,

$$
\sum_{j=1}^{N} \sum_{i}^{n_{j}} \tilde{q}_{f D j i}=\frac{1}{s}
$$

By solving equations (29) and (30), $m$ flow values and the pressure in the horizontal well can be obtained.

\section{Model Verification}

A semianalytical model proposed by Wang et al. [14] is compared with our model to validate. Some important parameters are as follows: homogeneous reservoir area $2500 \mathrm{~m} \times 2500 \mathrm{~m}$, reservoir permeability $k=0.01 \mathrm{md}$, fracture half-length $x_{f}=100 \mathrm{~m}$, fracture permeability $k_{f}=10 \mathrm{D}$, number of fractures formed by fracturing $N=5$, fracture spacing $200 \mathrm{~m}$, and horizontal well production $40 \mathrm{~m}^{3} / \mathrm{D}$. For the purpose of comparison with the homogeneous model, the permeability ratio of the inner region to the middle region and the permeability ratio of the inner region to the outer region in the proposed model are $\lambda_{21}=\lambda_{31}=1$; the storage ratio of the inner region to the middle region and the storage ratio of the inner region to the outer region are $\omega_{21}=\omega_{31}=1$; fracture conductivity $C_{\mathrm{fD}}=100$; the dimensionless drainage radius of the inner region, middle region, and outer region is $r_{e D 1}=5, r_{e D 2}=10$, and $r_{e D 3}=14.01$, respectively. The simulation validation result is shown in Figure 3. As shown in the figure, the result from the semianalytical model is in good agreement with the presented model, thus, verifying the reliability of the model result.

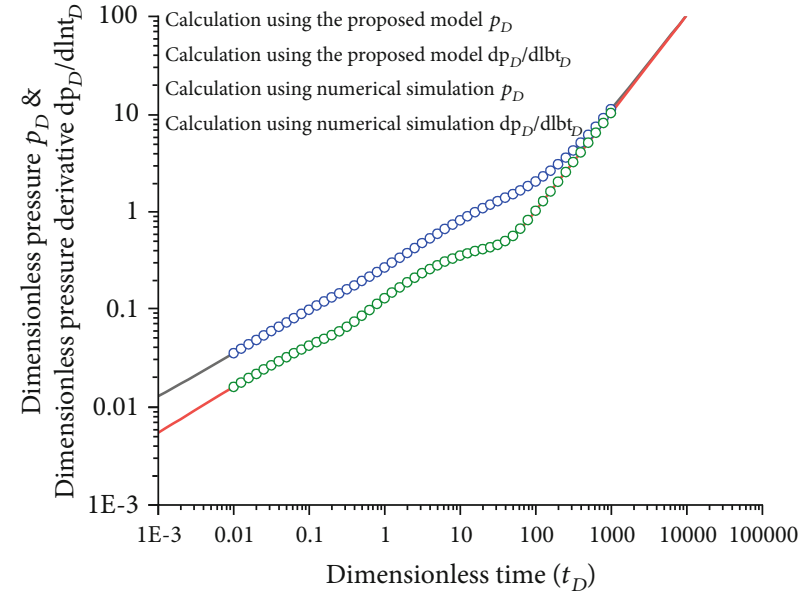

FIgURE 3: Comparative verification of the proposed model with the analytical model proposed by Wang et al. [14].

\section{Analysis and Application Example of Typical Curves}

4.1. Analysis of Flow Characteristics of a Multistage Fractured Horizontal Well in a Three-Region Composite Reservoir. The parameters in the proposed model for the multistage fractured horizontal well in the three-region reservoir are below: fracture conductivity $C_{\mathrm{fD}}=20$, dimensionless fracture spacing $L_{D}=8$, number of fractures $N=5$, permeability ratio of the inner region to the middle region $\lambda_{21}=2$, permeability ratio of the inner region to the outer region $\lambda_{31}=4$, storage ratio of the inner region to the middle region $\omega_{21}=2$, storage ratio of the inner region to the outer region $\omega_{31}=3$, included angle between the fracture and the $x$-axis $\theta=90 o$, and the dimensionless drainage radius of the inner region, middle region, and outer region $r_{e D 1}=100, r_{e D 2}=1000$, and $r_{e D 3}=5000$, respectively. The calculation result is shown in Figure 4. As shown in the figure, the flow in the multistage fractured horizontal well in the three-region composite reservoir can be divided into 10 stages based on the pressure derivative curves in Figure 4.

Stage 1. Bilinear flow stage: the pressure and pressure derivative curves show a straight line segment of 1/4 slope, respectively; the fluids near the reservoir flow perpendicularly to the fractures, while the fluids in the fractures flow perpendicularly to the horizontal wellbore.

Stage 2. Linear flow stage: the pressure and pressure derivative curves show a straight line segment of $1 / 2$ slope, respectively; the fluids flowing linearly near the reservoir mainly flow towards the hydraulic fractures in this stage.

Stage 3. The first radial flow stage: in this stage, a straight line of a constant value $1 / 2 \mathrm{~N}$ is shown on the pressure derivative curve. The number of fractures $N=5$, so a straight line of a constant value 0.1 is shown on the derivative curve.

Stage 4. Linear flow stage in the inner region: the pressure derivative curve shows a straight line segment of $1 / 2$ slope; 


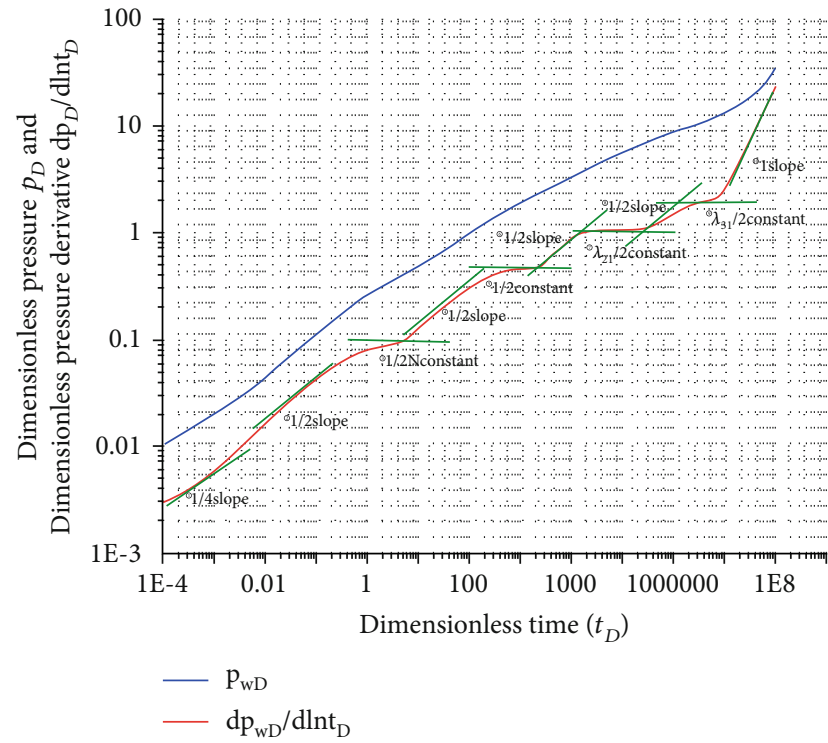

FIGURE 4: Verification of the model for the multistage fractured horizontal well in the three-region composite reservoir.

the fluids in the inner region flow perpendicularly to all fractures in this stage.

Stage 5. Radial flow stage in the inner region: a straight line segment of a constant value 0.5 is shown on the pressure derivative curve. As the pressure wave is propagated farther and farther, multiple hydraulic fractures appear to be a whole. From the perspective of the entire reservoir, radial flow in all hydraulic fractures occurs, and the distant fluids begin to converge towards the direction of multiple hydraulic fractures.

Stage 6. Linear flow stage in the middle region: the pressure derivative curve shows a straight line segment of $1 / 2$ slope; the fluids in the middle region flow linearly in this stage.

Stage 7. Radial flow stage in the middle region: in this stage, a straight line of a constant value $\lambda_{21} / 2$ is shown on the pressure derivative curve. The permeability ratio $\lambda_{21}$ of the inner region to the middle region is equal to 2 , so a straight line of a constant value 1 is shown on the derivative curve.

Stage 8. Linear flow stage in the outer region: the pressure derivative curve shows a straight line segment of $1 / 2$ slope; the fluids in the outer region flow linearly in this stage.

Stage 9. Radial flow stage in the outer region: in this stage, a straight line of a constant value $\lambda_{31} / 2$ is shown on the pressure derivative curve. The permeability ratio $\lambda_{31}$ of the inner region to the outer region is equal to 4 , so a straight line of a constant value 2 is shown on the derivative curve.

Stage 10. Boundary dominated flow stage: in this stage, the pressure derivative curve shows a straight line segment of unit slope; this stage symbolizes that the reservoir energy will be gradually depleted.
4.2. Analysis of Influential Factors. The parameters in the proposed model for the multistage fractured horizontal well in the three-region reservoir are below: dimensionless fracture spacing $L_{D}=8$, number of fractures $N=5$, permeability ratio of the inner region to the middle region $\lambda_{21}=2$, permeability ratio of the inner region to the outer region $\lambda_{31}=4$, storage ratio of the inner region to the middle region $\omega_{21}=2$, storage ratio of the inner region to the outer region $\omega_{31}=3$, fracture conductivity $C_{\mathrm{fD}}=20$, the dimensionless drainage radius of the inner region, middle region, and outer region $r_{e D 1}=100$, $r_{e D 2}=1000$, and $r_{e D 3}=5000$, respectively, and the included angle between the fracture and the $x$-axis $\theta=90^{\circ}$.

Figure 5(a) shows the influence of fracture conductivity on the pressure and pressure derivative curves, where the conductivity $C_{\mathrm{fD}}$ is set to 20,50 , and 300 , respectively. As shown in the figure, the fracture conductivity mainly affects the early bilinear flow stage of fractures. The larger the conductivity, the lower the dimensionless pressure, the smaller the pressure drop, and the smaller the pressure loss. As the conductivity increases, the early bilinear flow time becomes short. When fracture conductivity is $\geq 300$, the bilinear flow stage is replaced by the linear flow stage.

Figure 5(b) shows the storage coefficient of fracture spacing on the pressure and pressure derivative curves, where the fracture spacing $L_{D}$ is set to 8,4 , and 2, respectively. As shown in the figure, the larger the fracture spacing, the smaller the pressure loss. The fracture spacing mainly affects the radial flow in the early stage of fractures and the radial flow in the inner region. The smaller the fracture spacing, the longer the time of the radial flow in fractures and the inner region and the shorter the time of the linear flow in the reservoir in the early stage.

Figure 5(c) shows the radius ratio of the middle region to the inner region on the pressure and pressure derivative curves, where the radius ratio $r_{e D 2} / r_{e D 1}$ is set to 10,5 , and 2, respectively. As shown in the figure, the smaller the radius ratio of the middle region to the inner region, the smaller the dimensionless pressure and the smaller the pressure loss. As can be seen in the pressure derivative curve, the radius ratio of the middle region to the inner region mainly affects the duration of the radial flow in the middle region. As the radius ratio of the middle region to the inner region decreases, the time of the radial flow in the middle region gradually decreases until disappearance, and the time of the radial flow in the inner region is extended accordingly.

Figure 5(d) shows the radius ratio of the outer region to the middle region on the pressure and pressure derivative curves, where the radius ratio $r_{e D 3} / r_{e D 2}$ is set to 8,5 , and 2 , respectively. As shown in the figure, the smaller the radius ratio of the outer region to the middle region, the larger the late dimensionless pressure and the larger the pressure loss. As can be seen in the pressure derivative curve, the radius ratio of the outer region to the middle region mainly affects the duration of the radial flow in the outer region. As the radius ratio of the outer region to the middle region decreases, the time of the radial flow in the outer region gradually decreases until disappearance, and the time of pseudosteady state flow is reduced accordingly. 


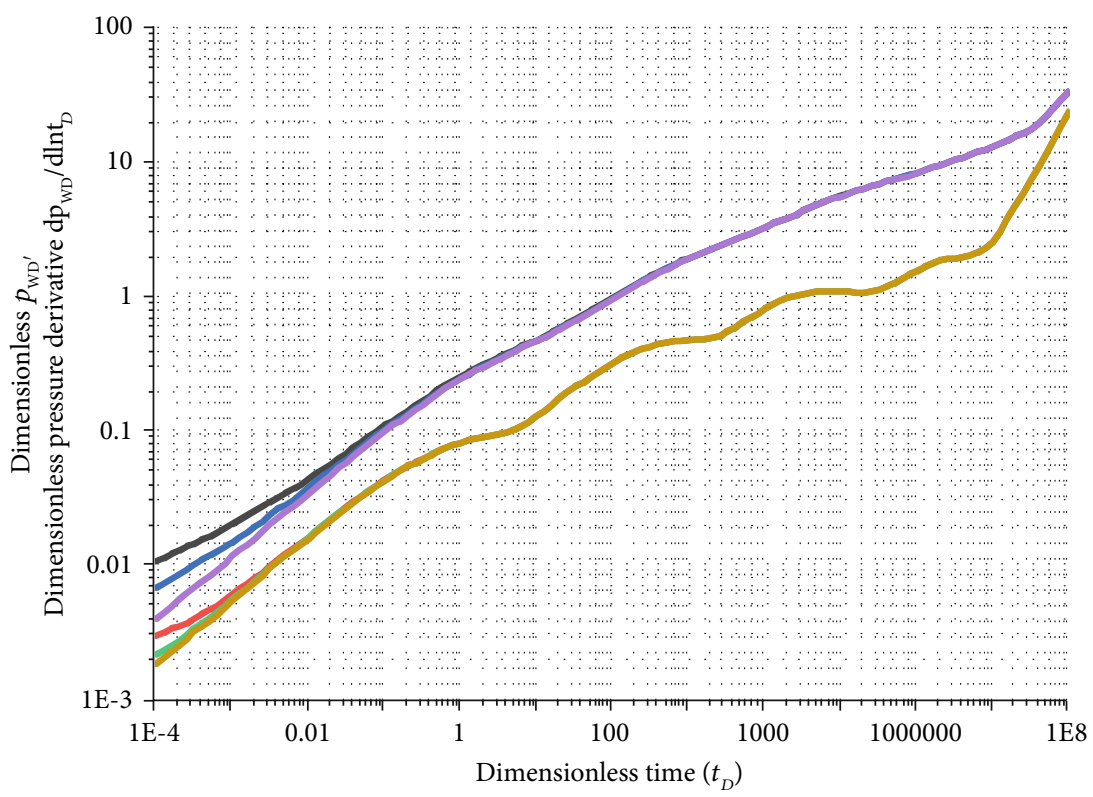

$$
\begin{array}{ll}
-p_{\mathrm{WD}} C_{\mathrm{fD}}=20 & -\mathrm{dp}_{\mathrm{WD}} / \mathrm{d} \ln t_{D} C_{\mathrm{fD}}=50 \\
-\mathrm{dp}_{\mathrm{WD}} / \mathrm{d} \ln t_{D} C_{\mathrm{fD}}=20 & -p_{\mathrm{WD}} C_{\mathrm{fD}}=300 \\
-p_{\mathrm{WD}} C_{f D}=50 & -\mathrm{dp}_{\mathrm{WD}} / \mathrm{dln}_{D} C_{\mathrm{fD}}=300
\end{array}
$$

(a)

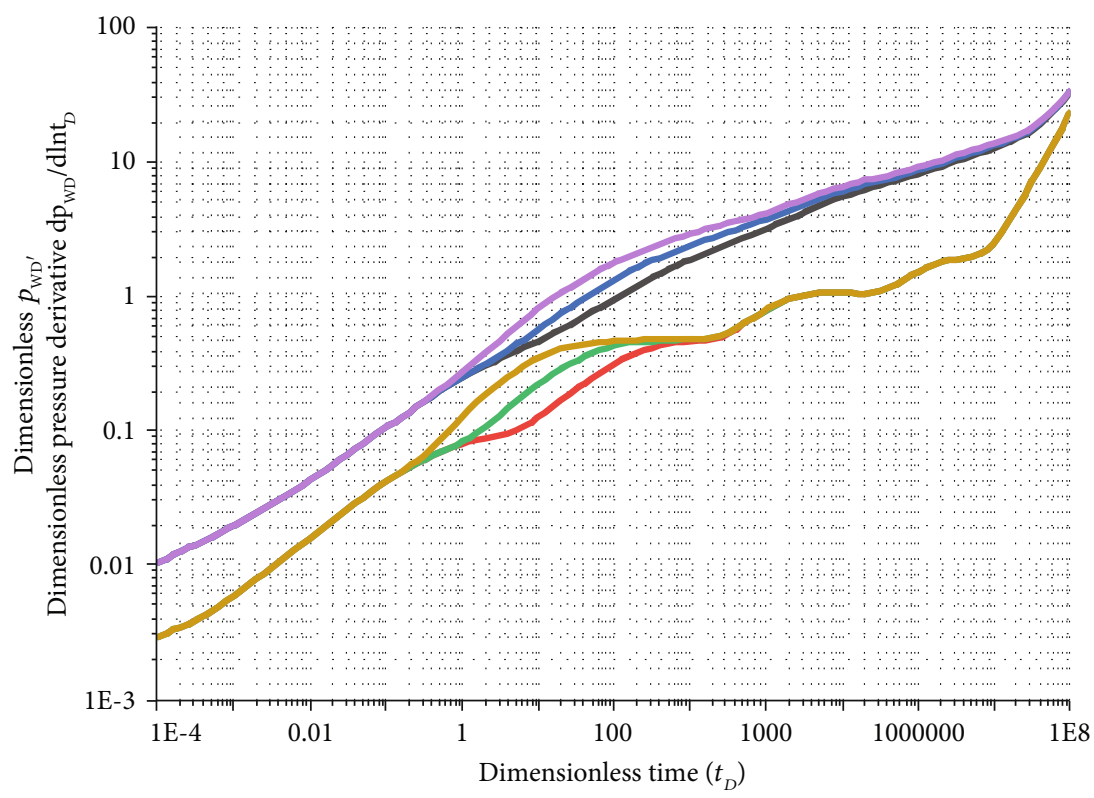

$$
\begin{aligned}
& -p_{W D} L_{D}=8 \\
& -\mathrm{dp}_{W D} / \mathrm{d} \ln t_{D} L_{D}=8 \\
& -p_{W D} L_{D}=4
\end{aligned}
$$

$$
\begin{aligned}
& -\mathrm{dp}_{W D} / \mathrm{dln}_{D} L_{D}=4 \\
& -p_{\mathrm{WD}} L_{D}=2 \\
& -\mathrm{dp}_{\mathrm{WD}} / \mathrm{dln}_{D} L_{D}=2
\end{aligned}
$$

(b)

Figure 5: Continued. 


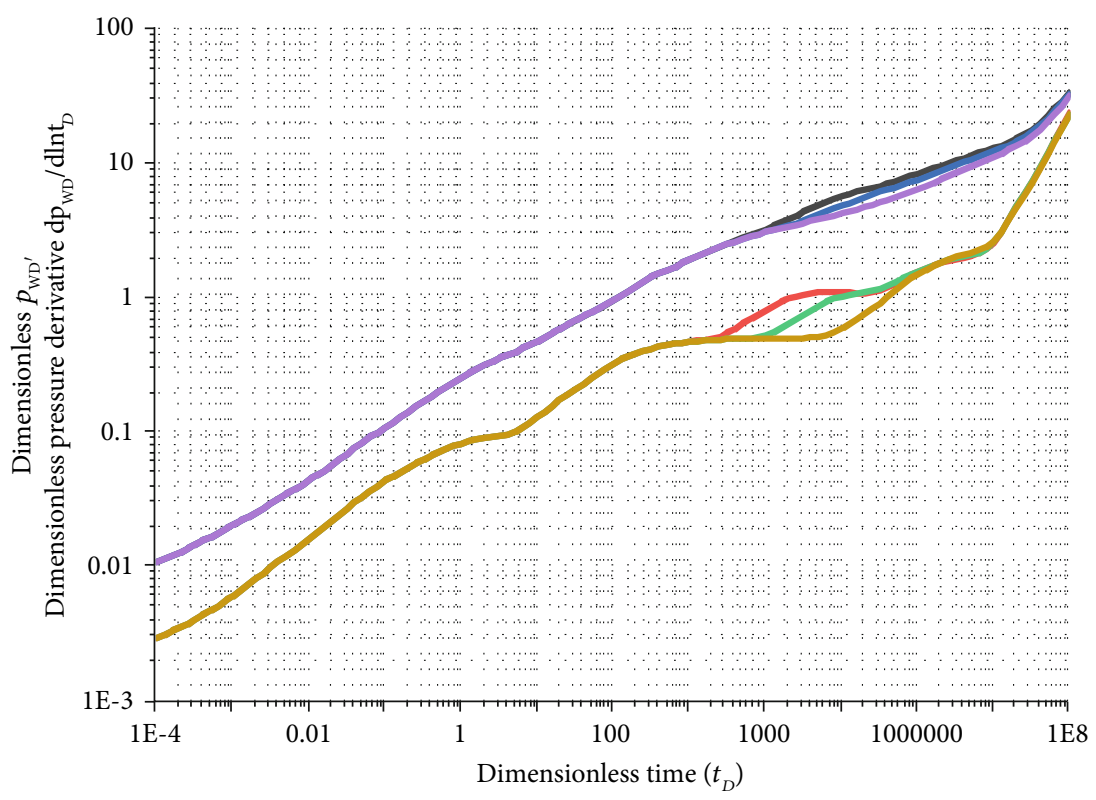
$-p_{\mathrm{WD}} r_{\mathrm{eD} 2} / r_{\mathrm{eD} 1}=10$
$-\mathrm{dp}_{\mathrm{WD}} / \mathrm{dln} t_{D} r_{\mathrm{eD} 2} / r_{\mathrm{eD} 1}=5$
$-\mathrm{dp}_{\mathrm{WD}} / \mathrm{dln} t_{\mathrm{D}} r_{\mathrm{eD} 1} / r_{\mathrm{eD} 2}=10$
$-p_{\mathrm{WD}} r_{\mathrm{eD} 2} / r_{\mathrm{eD} 1}=2$
- $p_{\mathrm{WD}} r_{\mathrm{eD} 2} / r_{\mathrm{eD} 1}=5$
$-\mathrm{dp}_{\mathrm{WD}} / \mathrm{d} \ln t_{\mathrm{D}} r_{\mathrm{eD} 2} / r_{\mathrm{eD} 1}=2$

(c)

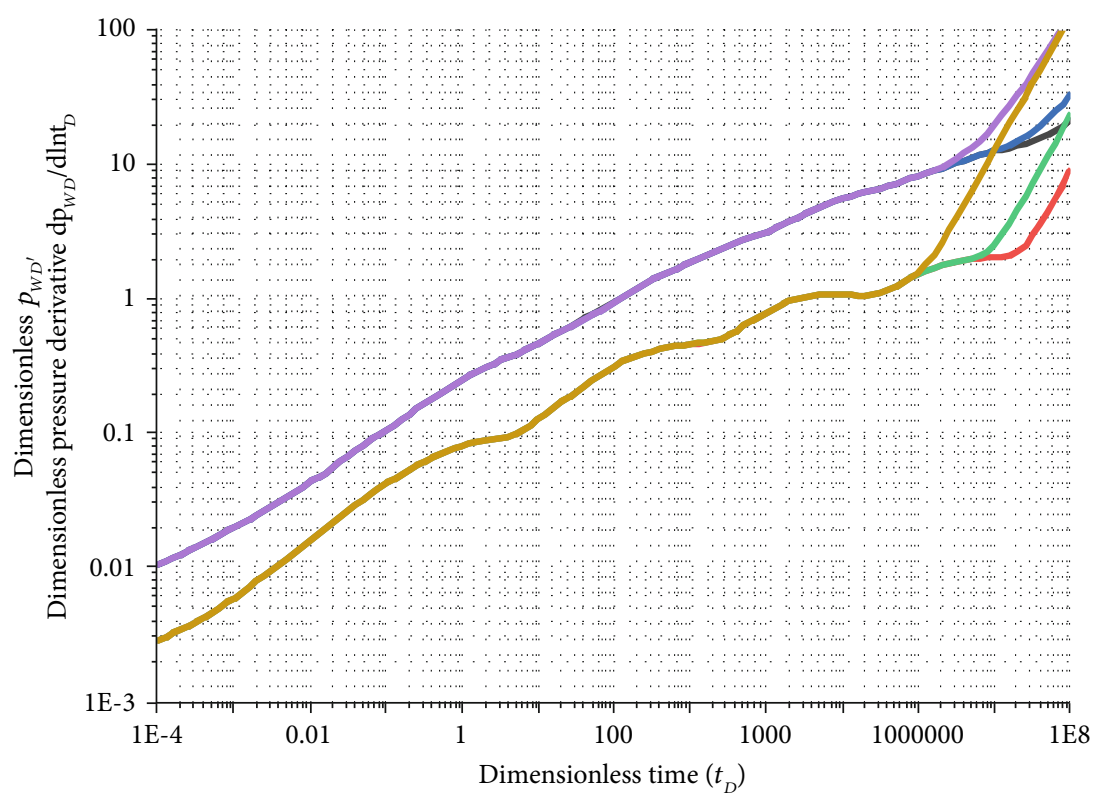

$$
\begin{aligned}
& -p_{\mathrm{WD}} r_{\mathrm{eD} 3} / r_{\mathrm{eD} 2}=8 \\
& -\mathrm{dp}_{\mathrm{WD}} / \mathrm{d} \ln t_{\mathrm{D}} r_{\mathrm{eD} 3} / r_{\mathrm{eD} 2}=8 \\
& -p_{\mathrm{WD}} r_{\mathrm{eD} 3} / r_{\mathrm{eD} 2}=5
\end{aligned}
$$$$
-\mathrm{dp}_{\mathrm{WD}} / \mathrm{d} \ln t_{\mathrm{D}} r_{\mathrm{eD} 3} / r_{\mathrm{eD} 2}=5
$$$$
p_{W D} r_{\mathrm{eD} 3} / r_{\mathrm{eD} 2}=2
$$$$
-\mathrm{dp}_{W D} / \mathrm{d} \ln t_{D} r_{\mathrm{eD} 3} / r_{\mathrm{eD} 2}=2
$$

(d)

Figure 5: Continued. 


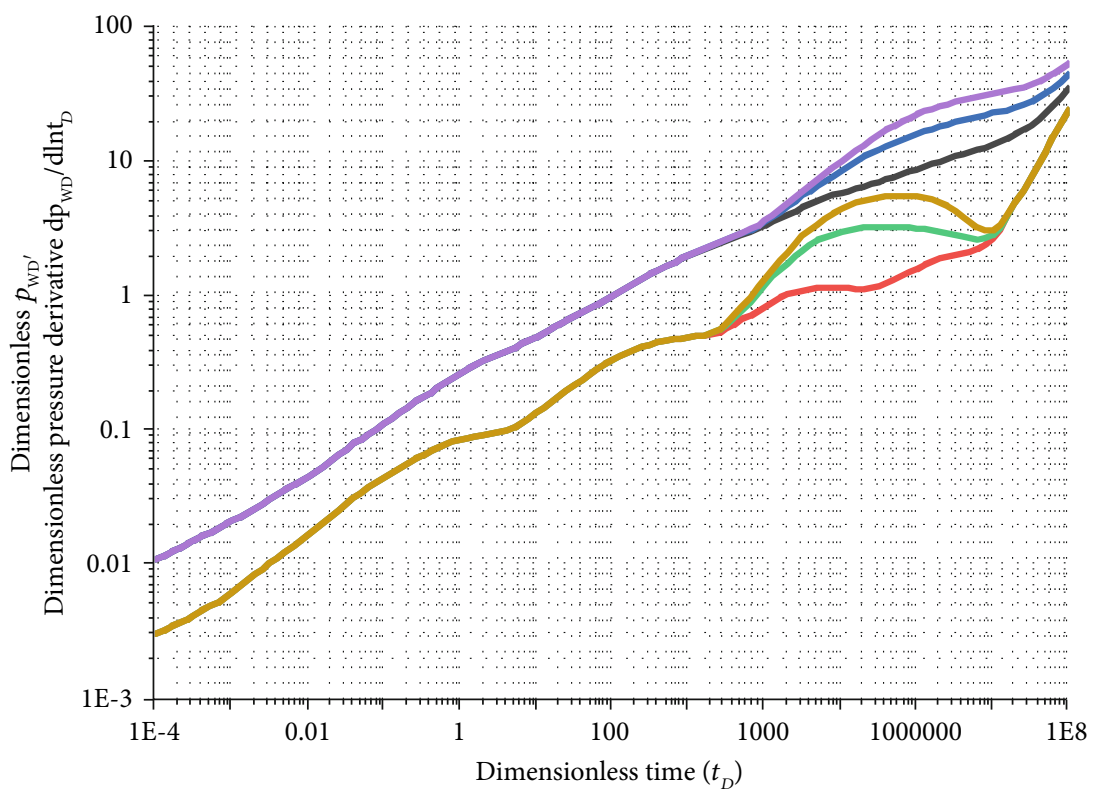

$$
\begin{array}{ll}
-p_{\mathrm{WD}} \lambda_{21}=2 & -\mathrm{dp}_{\mathrm{WD}} / \mathrm{dlnt}_{D} \lambda_{21}=6 \\
-\mathrm{dp}_{\mathrm{WD}} / \mathrm{dlnt}_{\mathrm{D}} \lambda_{21}=2 & -p_{\mathrm{WD}} \lambda_{21}=10 \\
-p_{\mathrm{WD}} \lambda_{21}=6 & -\mathrm{dp}_{\mathrm{WD}} / \mathrm{dlnt}_{D} \lambda_{21}=10
\end{array}
$$

(e)

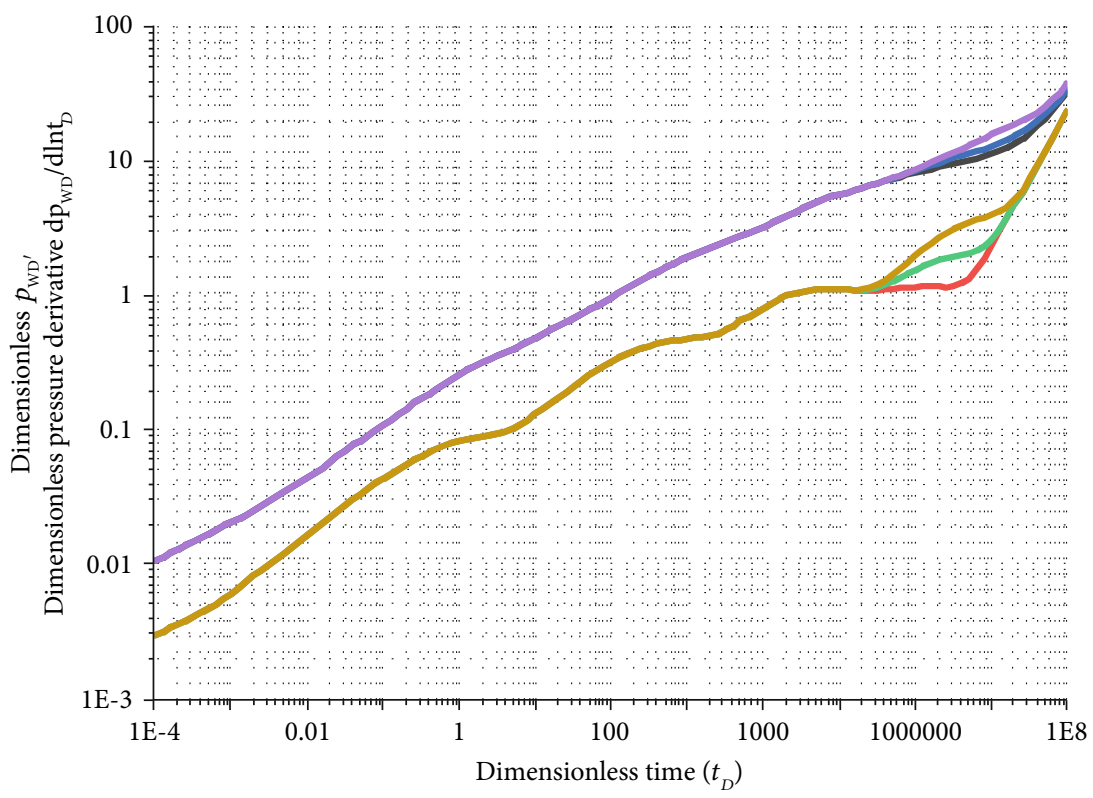

$$
\begin{aligned}
& -p_{\mathrm{WD}} \lambda_{31}=2 \\
& -\mathrm{dp}_{\mathrm{WD}} / \mathrm{dlnt}_{\mathrm{D}} \lambda_{31}=2 \\
& -p_{\mathrm{WD}} \lambda_{31}=4
\end{aligned}
$$$$
-\mathrm{dp}_{\mathrm{wD}} / \mathrm{dlnt}_{D} \lambda_{31}=4
$$$$
\text { - } p_{\mathrm{WD}} \lambda_{31}=8
$$$$
-\mathrm{dp}_{\mathrm{wD}} / \mathrm{dlnt}_{D} \lambda_{31}=8
$$

(f)

Figure 5: Continued. 


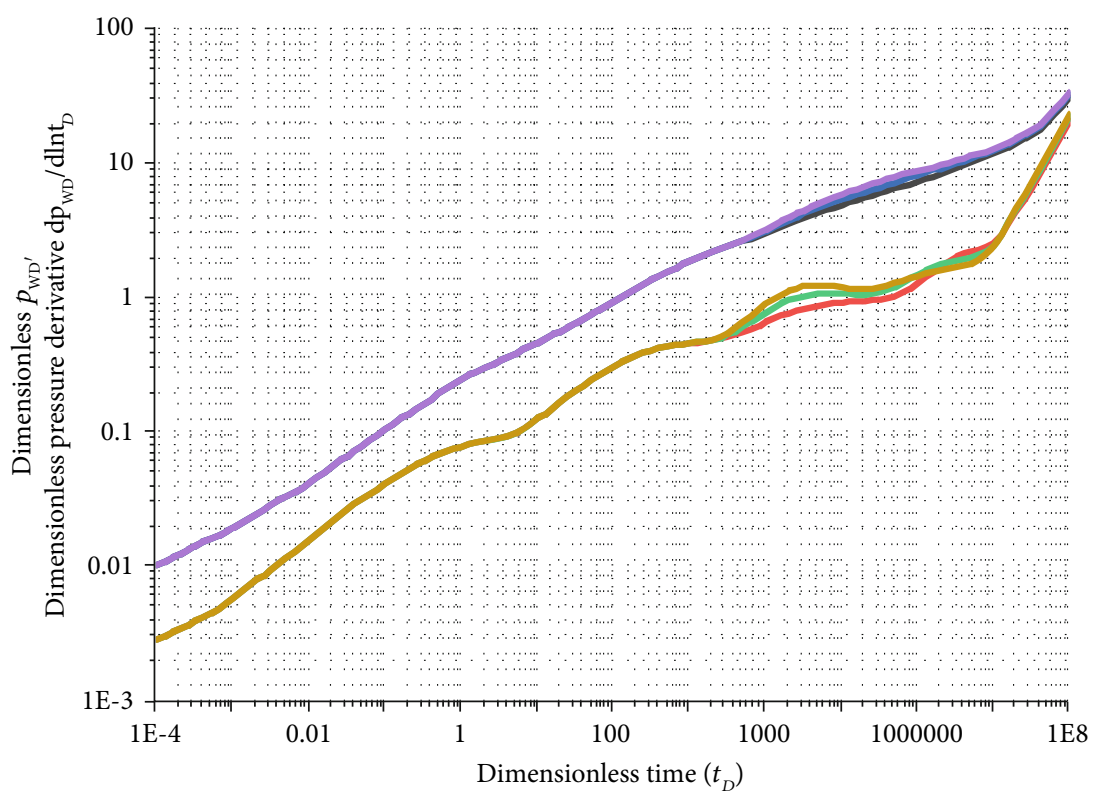

$$
\begin{aligned}
& -p_{\mathrm{WD}} \omega_{21}=1 \\
& -\mathrm{dp}_{\mathrm{WD}} / \mathrm{dlnt}_{D} \omega_{21}=1 \\
& -p_{\mathrm{WD}} \omega_{21}=2
\end{aligned}
$$

$-\mathrm{dp}_{\mathrm{WD}} / \mathrm{dlnt}_{D} \omega_{21}=2$

$-p_{\mathrm{WD}} \omega_{21}=4$

$-\mathrm{dp}_{\mathrm{WD}} / \mathrm{dlnt}_{D} \omega_{21}=4$

(g)

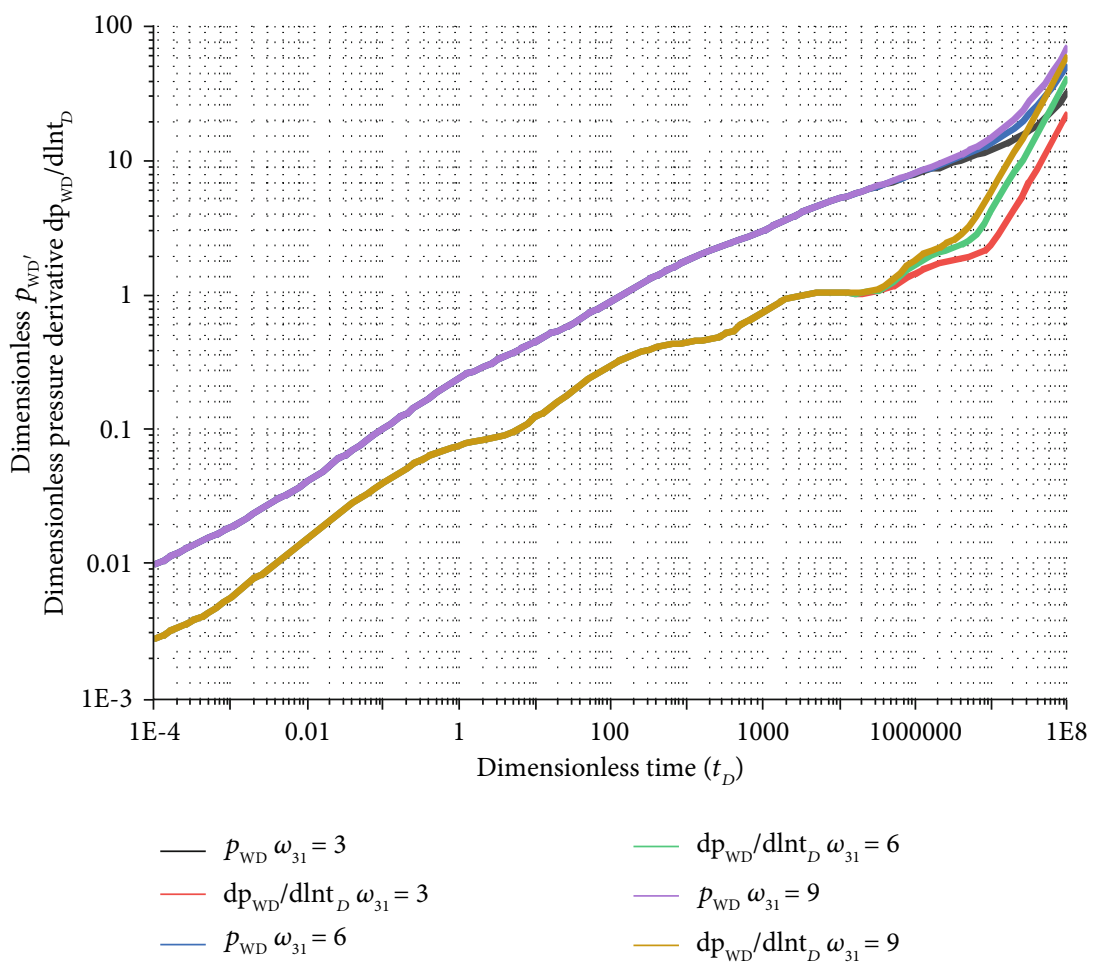

(h)

Figure 5: Analysis of influential factors for the multistage fractured horizontal well in the three-region reservoir: (a) influence of fracture conductivity $C_{\mathrm{fD}}$ on the typical curves; (b) influence of fracture spacing $L_{D}$ on the typical curves; (c) influence of the dimensionless radius ratio of the middle region to the inner region on the typical curves; (d) influence of the dimensionless radius ratio of the outer region to the middle region on the typical curves; (e) influence of the permeability ratio $\lambda_{21}$ of the inner region to the middle region on the typical curves; (f) influence of the permeability ratio $\lambda_{31}$ of the inner region to the outer region on the typical curves; ( $g$ ) influence of the storage ratio $\omega_{21}$ of the inner region to the middle region on the typical curves; (h) influence of the storage ratio $\omega_{31}$ of the inner region to the outer region on the typical curves. 


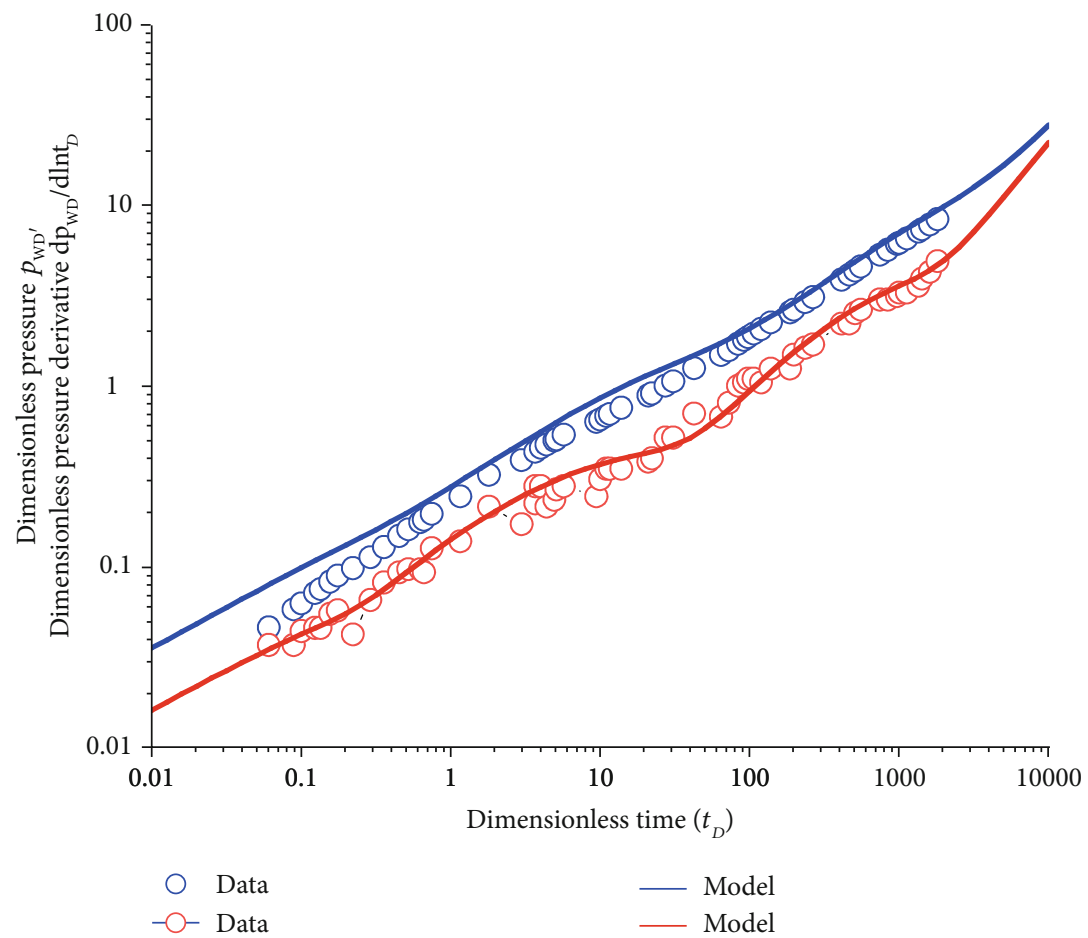

FiguRE 6: Example of fitting between the model for the multistage fractured horizontal well in the three-region reservoir and the production data.

Figure 5(e) shows the permeability ratio of the inner region to the middle region on the pressure and pressure derivative curves, where the permeability ratio $\lambda_{21}$ is set to 2,6 , and 10 , respectively. As shown in the figure, the larger the permeability ratio of the inner region to the middle region, the larger the late dimensionless pressure and the larger the pressure loss. As can be seen in the pressure derivative curve, the permeability ratio of the inner region to the middle region mainly affects the flow characteristics of the middle region. When the permeability ratio $\lambda_{21}$ of the inner region to the middle region is set to 2,6 , and 10 , respectively, a constant value of 1,3 , and 5 is, respectively, shown on the dimensionless pressure derivative curve. As the permeability ratio of the inner region to the middle region increases, the duration of the radial flow in the outer region is reduced.

Figure 5(f) shows the permeability ratio of the inner region to the outer region on the pressure and pressure derivative curves, where the permeability ratio $\lambda_{31}$ is set to 2,4 , and 8 , respectively. As shown in the figure, the larger the permeability ratio of the inner region to the outer region, the larger the late dimensionless pressure and the larger the pressure loss. As can be seen in the pressure derivative curve, the permeability ratio of the inner region to the outer region mainly affects the flow characteristics of the outer region. As the permeability ratio of the inner region to the outer region decreases, the constant value of the radial flow in the outer region is reduced. When the permeability of the middle region is equal to that of the outer region $\left(\lambda_{31}=\lambda_{21}=2\right)$, the inner region and the outer region are equivalent to a region, and the derivative curve shows a unit constant value.

Figure 5(g) shows the storage ratio of the inner region to the middle region on the pressure and pressure derivative curves, where the storage ratio $\omega_{21}$ is set to 1,2 , and 4 , respectively. As shown in the figure, the larger the storage ratio of the inner region to the middle region, the larger the late dimensionless pressure and the larger the pressure loss, but the variation is not very remarkable. As can be seen in the pressure derivative curve, the storage ratio of the inner region to the middle region mainly affects the characteristics of medium-term linear flow and medium-term radial flow; as the storage ratio increases, the medium-term linear flow deviates from the straight line segment of $1 / 2$ slope.

Figure $5(\mathrm{~h})$ shows the storage ratio of the inner region to the outer region on the pressure and pressure derivative curves, where the storage ratio $\omega_{31}$ is set to 3,6 , and 9 , respectively. As shown in the figure, the larger the storage ratio of the inner region to the outer region, the larger the late dimensionless pressure and the larger the pressure loss. As can be seen in the pressure derivative curve, the storage ratio of the inner region to the outer region mainly affects the characteristics of late linear flow, radial flow, and pseudosteady state flow; as the storage ratio increases, the linear flow in the outer region deviates from the straight line segment of $1 / 2$ slope, and the radial flow deviates from the constant and enters the pseudosteady state flow stage ahead of time.

4.3. Example Analysis. Through fitting of the site-measured data of pressure and pressure derivative based on the model in the paper, the parameters of a reservoir after multistage fracturing of a horizontal well can be obtained as follows: inner region permeability $84.8 \times 10^{-3} \mu \mathrm{m}^{2}$, middle region permeability $6.1 \times 10^{-3} \mu \mathrm{m}^{2}$, outer region permeability $13.05 \times 10^{-3} \mu \mathrm{m}^{2}$, fracture half-length $120 \mathrm{~m}$, fracture number 5 , oil viscosity $9.7 \mathrm{mPa} \cdot \mathrm{s}$, inner region radius $1524 \mathrm{~m}$, 
middle region radius $1869 \mathrm{~m}$, outer region radius $6081.6 \mathrm{~m}$, fracture spacing $216 \mathrm{~m}$, initial formation pressure $30 \mathrm{MPa}$, the storage ratio of the inner region to the middle region 10.67 , and the storage ratio of the inner region to the outer region 5.54. Figure 6 shows the result of the comparison between the actual production data and the model for the multistage fractured horizontal well in the three-region reservoir. According to the result, they tally with each other well, and the accuracy of the unsteady-state model presented in this paper for the multistage fractured horizontal well in the three-region reservoir has been verified.

\section{Conclusion}

(1) This paper has presented the model for the multistage fractured horizontal well in a three-region composite reservoir. The model takes account of the flow in both three-region composite media and hydraulic fractures. The semianalytical solution of the model has been obtained from Laplace transformation and point source integration

(2) The accuracy of the proposed model has been verified through the comparison with the numerical simulation result

(3) According to the analysis of flow characteristics, the flow stage is divided into ten stages below: bilinear flow stage: a straight line of $1 / 4$ slope is shown on the pressure derivative curve; linear flow stage: a straight line of $1 / 2$ slope is shown on the pressure derivative curve; the first radial flow stage: in this flow stage, a straight line segment of a constant value $1 / 2 \mathrm{~N}$ is shown on the pressure derivative curve; linear flow stage in the inner region: a straight line of $1 / 2$ slope is shown on the pressure derivative curve; radial flow stage in the inner region: a straight line of a constant value 0.5 is shown on the pressure derivative curve; linear flow stage in the middle region: a straight line of $1 / 2$ slope is shown on the pressure derivative curve; radial flow stage in the middle region: a straight line of a constant value $\lambda_{21} / 2$ is shown on the pressure derivative curve; linear flow stage in the outer region: a straight line of $1 / 2$ slope is shown on the pressure derivative curve; radial flow stage in the outer region: a straight line of a constant value $\lambda_{31} / 2$ is shown on the pressure derivative curve; boundary control flow stage: a straight line segment of unit slope is shown on the pressure derivative curve

(4) The influence of some reservoir parameters and fracture sensitivity parameters on the typical curves has been analyzed, mainly including fracture conductivity, fracture spacing, the radius ratio of the middle region to the inner region, the radius ratio of the outer region to the middle region, the permeability ratio of the inner region to the middle region, the permeability ratio of the inner region to the outer region, the storage ratio of the inner region to the middle region, and the storage ratio of the inner region to the outer region

(5) Finally, the accuracy of the presented model has been verified through the analysis of an example

\section{Data Availability}

All data generated or used during the study appear in the submitted article are available from the corresponding author upon request.

\section{Conflicts of Interest}

The authors declare that there is no conflict of interest regarding the publication of this paper.

\section{References}

[1] Z. Qiu, C. N. Zou, J. Z. Li, Q. L. Guo, X. Z. Wu, and L. H. Hou, "Unconventional petroleum resources assessment: progress and future prospects," Natural Gas Geoscience, vol. 24, no. 2, pp. 238-246, 2013.

[2] F. H. Zeng, J. C. Guo, Y. B. Xu, and Z. JZ, "Factors affecting production capacity of fractured horizontal wells," Petroleum Exploration and Development, vol. 34, no. 4, pp. 474-477, 2007.

[3] A. Gringarten, H. Ramey, and R. Raghavan, "Pressure analysis for fractured wells," in Paper 4051, presented at the SPE-AIME 47th Annual Fall Meeting, San Antonio, Texas, 1972.

[4] A. Gringarten, H. Ramey, and R. Raghavan, "Applied pressure analysis for fractured well," Journal of Petroleum Technology, vol. 27, pp. 887-892, 1975.

[5] H. Cinco-Ley, H. J. Ramey Jr., and F. Miller, "Unsteady-state pressure distribution created by a well with an inclined fracture," in Paper 5591 presented at the SPE 50th Annual Technical Conference and Exhibition, Dallas, 1975.

[6] L. H. Cinco, V. F. Samaniego, and A. N. Dominguez, "Transient pressure behavior for a well with a finite-conductivity vertical fracture," Society of Petroleum Engineers Journal, vol. 18, no. 4, pp. 253-264, 1978.

[7] C. Zhiming, L. Xinwei, H. Chenghui et al., "Productivity estimations for vertically fractured wells with asymmetrical multiple fractures," Journal of Natural Gas Science and Engineering, vol. 21, pp. 1048-1060, 2014.

[8] J. Guo, J. Zeng, X. Wang, and F. Zeng, "Analytical model for multi-fractured horizontal wells in heterogeneous shale reservoirs," in SPE Asia Pacific Oil \& Gas Conference and Exhibition, Perth, Australia, 2016.

[9] Y. He, S. Cheng, Z. Rui et al., "An improved rate-transient analysis model of multi-fractured horizontal wells with nonuniform hydraulic fracture properties," Energies, vol. 11, no. 2, p. 393, 2018.

[10] E. Ozkan, M. L. Brown, R. Raghavan, and H. Kazemi, "Comparison of fractured-horizontal-well performance in tight sand and shale reservoirs," SPE Reservoir Evaluation \& Engineering, vol. 14, no. 2, pp. 248-259, 2011.

[11] I. G. Brohi, M. Pooladi-Darvish, and R. Aguilera, "Modeling fractured horizontal wells as dual porosity composite reservoirs-application to tight gas, shale gas and tight oil cases," in SPE Western North American Region Meeting, Society of Petroleum Engineers, 2011. 
[12] E. Stalgorova and L. Mattar, "Practical analytical model to simulate production of horizontal wells with branch fractures," in SPE Canadian Unconventional Resources Conference, Calgary, Alberta, Canada, 2012.

[13] M. Brown, E. Ozkan, R. Raghavan, and H. Kazemi, "Practical solutions for pressure-transient responses of fractured horizontal wells in unconventional shale reservoirs," SPE Reservoir Evaluation \& Engineering, vol. 14, no. 6, pp. 663-676, 2011.

[14] J. Wang, X. Wang, and W. Dong, "Rate decline curves analysis of multiple-fractured horizontal wells in heterogeneous reservoirs," Journal of Hydrology, vol. 553, pp. 527-539, 2017.

[15] L. Tao, J. Guo, X. Zhou, A. Kitaeva, and J. Zeng, "A new productivity prediction hybrid model for multi-fractured horizontal wells in tight oil reservoirs," in SPE Russian Petroleum Technology Conference, Moscow, Russia, 2018.

[16] H. Ahmadi, C. R. Clarkson, H. Hamdi, and H. Behmanesh, "Analysis of communicating multi-fractured horizontal well production data using the dynamic drainage area concept," in SPE Canada Unconventional Resources Conference, 2020. 